\title{
Semantic constraints and judged preference for interpretations of ambiguous sentences
}

\author{
GREGG C. ODEN \\ University of Wisconsin, Madison, Wisconsin 53706
}

\begin{abstract}
It is proposed that the degree of sensibleness of sentences is determined by semantic constraints which may be more or less satisfied. Such continuous semantic constraints were examined in two experiments in which subjects judged the likelihood of obtaining each of the interpretations of ambiguous sentences. The sentences were factorially generated by independently varying the degree to which semantic constraints for each interpretation were satisfied. In one experiment, the semantic constraints were manipulated by varying critical words within the ambiguous sentence; in the other experiment, a preceding context sentence was used. The results of both experiments supported the hypotheses that the judged likelihood was a direct function of the relative sensibleness of the interpretations, that semantic constraints determined the degree of sensibleness of each interpretation, and that these semantic constraints are continuous restrictions which are independent of each other and stable from sentence to sentence in which they occur.
\end{abstract}

It has become increasingly apparent that much of human knowledge is "fuzzy" rather than discrete in nature: Many subjective categories are fuzzy sets (Zadeh, $1965)$ to which objects may belong to varying degrees (e.g., Hersh \& Caramazza, 1976; Oden, 1977; Rosch, $1973,1975)$ and many semantic propositions are fuzzy logical expressions (Zadeh, 1974) which may be more or less true (e.g., Oden, in press; Oden \& Hogan, Note 1). For example, the categories of fish, furniture, and rich people are fuzzy sets in that there are some things which definitely belong to them, some things which "sort of" belong, and so on. Similarly, propositions such as "Rich people drive big cars" are not absolutely true or absolutely false but, rather, true to some intermediate degree.

Subjective categories and propositions are the fundamental building blocks of most current models of semantic knowledge (e.g., Anderson, 1976; Kintsch, 1974; Norman \& Rumelhart, 1975). Therefore, the introduction of fuzziness at this level will have ramifications for many, if not all, higher level semantic

This paper is partially based on a $\mathrm{PhD}$ dissertation submitted to the University of California, San Diego. The author thanks his graduate advisor, Norman Anderson, for much help, encouragement, and good advice. Thanks also to dissertation committee members Donald Norman, David Rumelhart, Edward Klima, and Timothy Smith, to James Levin, Lola Lopes, and Dominic Massaro for their helpful discussions, and to Valerie Hans and Bo Parker for running the subjects. This work was supported by National Science Foundation Grants GB-21028 and GB-36918 to Norman H. Anderson and by Wisconsin Alumni Research Foundation Grant, Project 150372, to the author, and was facilitated by National Institute of Mental Health support of the Center for Human Information Processing. Reprint requests should be sent to: Gregg Oden, Department of Psychology, University of Wisconsin, Madison, Wisconsin 53706. phenomena. The present paper focuses on the semantic constraints in language and examines whether these constraints should also be considered fuzzy, that is, considered to be constraints that may be more or less satisfied.

Intuitively, a semantic constraint is a relationship between two parts of a proposition such that the meaning of one part constrains what the other part may be, or in other words, it is a limitation on the ways in which particular semantic elements may be sensibly related. A somewhat more formal definition, but one which specifically allows for constraints to be more or less satisfied, is: a semantic constraint is a function that is associated with a particular semantic relationship and that defines, for each combination of semantic elements that may enter into the relationship, the degree of sensibleness of the resulting semantic structure. This definition requires that sensibleness be considered a continuous variable, whereas it has traditionally been thought of as taking on only two values: sense and nonsense. Thinking of sensibleness as a continuous quality would, however, also seem to be a natural extension of considering sets and propositions to be nondiscrete. Furthermore, the notion of continuous sensibleness is not entirely novel. For example, Lakoff (1972) has argued for the continuousness of sensibleness on linguistic grounds and has observed that there is even a "hedge" in English that is based on the existence of intermediate degrees of sensibleness: "To the extent that it makes sense to say that...."

Semantic constraints, as they are defined above, are meant to include what might more specifically be called pragmatic constraints. Semantic knowledge is that which is believed to be true by the definition of what things are, whereas pragmatic knowledge is that 
which is believed to be true simply because things happen to be that way. However, it is the content of a given piece of knowledge that constrains what other things may be true, independent of the grounds upon which that knowledge is based. Therefore, the present paper will examine constraints that are based on world knowledge of all kinds, whether pragmatic or more strictly semantic, and the term "semantic" will be used in the broader sense that includes pragmatic in much the same way that it is used in the phrase "semantic memory."

Continuous semantic constraints have previously been studied by Oden and Anderson (1974), who examined how the effects of multiple constraints within a sentence are combined to determine the total sensibleness of the sentence. Oden and Anderson asked subjects to judge the likelihood of events described by agent-verb-recipient propositions, for which the compatibility between the agent and the verb and between the recipient and the verb were independently manipulated. The results supported the hypothesis that, in general, the separate constraints are evaluated independently and the resulting sensibleness values are then combined by a multiplying rule. In the cases where this is true, complex semantic constraints holding over a large part of a sentence may be decomposed into a simple function of more primitive constraints, rather than having to be specified separately.

There were, however, a few cases in the Oden \& Anderson (1974) study that were exceptions to this general result. That is, certain agent-verb-recipient combinations were found whose joint effect on the sensibleness of a sentence was configural, in that it could not be decomposed into a multiplicative function of the effects of the pairwise constraints. However, these configural effects appeared to be due to specific higher order three-way constraints, such as that bashful people are especially shy about socializing with particularly attractive people. Thus, taken together, the results of this study provide support for the hypothesis that people use continuous semantic constraints to determine the degree of sensibleness of sentences, but that not all complex semantic constraints are decomposable.

The present experiments extend the study of continuous semantic constraints through the use of ambiguous sentences. In these experiments, subjects judged which interpretation of an ambiguous sentence they would be likely to obtain as the meaning of the sentence and how much more likely it seemed than the alternative interpretation. It was assumed that subjects would prefer the interpretation which was most sensible and that, in fact, the degree of preference for that interpretation would be a direct function of the relative sensibleness of the two interpretations. Consequently, this task seems ideally suited to the study of semantic constraints, since it is both a natural linguistic problem for people to solve and a task that allows the semantic constraints of one interpretation to be "pitted" against those of the other interpretation. In this way it is possible to determine if continuous semantic constraints are psychologically isolable, stable relations that play a constant role from sentence to sentence in which they occur. If semantic constraints are psychologically independent, then fixing all of the words involved in constraints that affect one interpretation should result in a constant sensibleness value for that interpretation, even if the sensibleness of the other interpretation is varied as its constraints are made to be more or less satisfied. With the ambiguous sentence judgment task, this may be determined even for many higher order or configural semantic constraints.

In addition, the question of how people decide which interpretation they would be more likely to obtain from an ambiguous sentence is an interesting problem in its own right. For example, this task of judging the relative likelihoods of ambiguous sentence interpretations is sometimes used (e.g., Olson \& Mackay, 1974) to tap subjects' linguistic intuitions about the degree of imbalance or bias of the ambiguous sentences. The bias of an ambiguous sentence is the degree to which one interpretation is more strongly preferred than the alternative. This variable has been repeatedly shown (e.g., Cairns, 1973; Holmes Arwas, \& Garrett, 1977; MacKay, 1970; Olson \& MacKay, 1974) to be very important for language processing. Therefore, it would be valuable to know if the bias of an ambiguous sentence is, in fact, determined by continuous semantic constraints governing its interpretations, and if so, exactly how bias relates to the sensibleness of the alternative interpretations.

It was hypothesized that subjects' judgments of the relative likelihood of obtaining one interpretation as opposed to the alternative would be a direct function of the relative sensibleness. Specifically, it was hypothe. sized that subjects' judgments in the present experiments would follow a simple algebraic rule:

$$
L\left(I_{1}\right)=\frac{S\left(I_{1}\right)}{S\left(I_{1}\right)+S\left(I_{2}\right)},
$$

in which the judged relative likelihood of obtaining the first interpretation, $\mathrm{L}\left(\mathrm{I}_{1}\right)$, is equal to the sensibleness of that interpretation, $S\left(I_{1}\right)$, divided by the sum of the sensibleness values.

To fully test the proposed judgment rule and whether semantic constraints are psychologically independent entities requires that constraints be found for each interpretation that affect only the interpretation and that may be manipulated in ways that do not in addition disturb either syntactic constraints or any other semantic constraints that may affect the other interpretation. This can be done, but it demands care in stimulus construction, since most words in a sentence 
are involved in several semantic and syntactic constraints that affect the sensibleness and grammaticality of all interpretations. However, this is a fact about the complexity of linguistic stimuli rather than a conceptual shortcoming of the judgment rule or of the formulation of semantic constraints and, fortunately, it is also not an insurmountable problem

One way in which it is possible to independently manipulate semantic constraints for the two interpretations involves sentences with surface-structure ambiguities. For example, the sentence: "The girl saw the boy with the binoculars," is ambiguous due to the fact that the prepositional phrase may be a constituent of the direct object noun phrase or it may be immediately dominated by the verb phrase node in the surface structure. The semantic structures of these two interpretations are diagrammed in Figure 1 in roughly the representation of Norman and Rumelhart (1975). The first interpretation given in Figure 1 is that the girl used binoculars to see the boy, and the second is that the girl saw a boy, who happened to have binoculars. As can be seen by comparing the two diagrams in Figure 1, most of the semantic relationships are the same for the two interpretations and consequently, according to the present formulation, most of the semantic constraints will also be shared.

The one semantic relationship that is a part of the first interpretation and not of the second is the instrument relationship holding between "binoculars" and the verb. Thus, by changing the verb, it should be possible to decrease the compatibility of binoculars as an instrument because of the semantic constraints

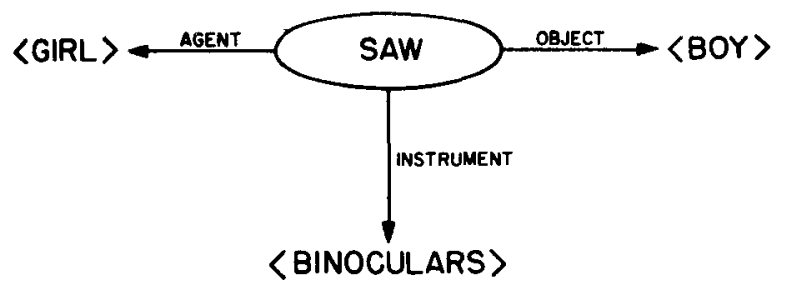

INTERPRETATION ।

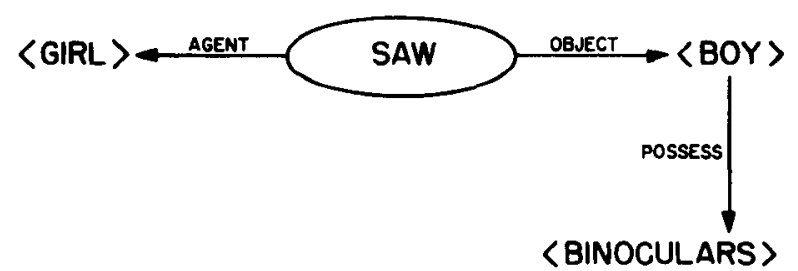

INTERPRETATION 2

Figure 1. Semantic structures of the two main interpretations of the sentence "The girl saw the boy with the binoculars." associated with that relationship. For example, changing the verb to "touched": "The girl touched the boy with the binoculars," seems to make it less reasonable for the binoculars to be the instrument of the action and, therefore, causes Interpretation 1 to be less sensible. Furthermore, since it is still perfectly reasonable for the girl to be the agent of "touched" and the boy to be the object, this manipulation appears to only affect the one critical semantic constraint that is not relevant to Interpretation 2 . This, of course, would not be true for every verb, but all that is needed for the present experiment is that stimuli be constructed using verbs that satisfy this requirement.

Similarly, only Interpretation 2 includes the possessive relationship between the object and the binoculars. The associated semantic constraint may be manipulated by changing the object, for example, to "dog": "The girl saw the dog with the binoculars," which seems to make it less likely that the object has the binoculars in his possession. Once again, while there are many different nouns that could be used as the object in order to manipulate the critical semantic constraint for Interpretation 2, it is also important to use only those words that satisfy all of the other semantic and syntactic constraints that govern either interpretation. In particular, it is necessary that every word selected also be appropriate as the object for each of the verbs that are to be used to vary the sensibleness of Interpretation 1. In this example, "dog" satisfies this requirement, since it is an appropriate object for both "saw" and "touched." In contrast, a word such as "amoeba" clearly should not be used.

The first experiment used this technique of changing words in sentences that are surface-structurally ambiguous in order to factorially vary the degree to which the hypothesized semantic constraints of each interpretation are satisfied. This allows the proposed judgment rule and, more importantly, the independence and stability of the semantic constraints to be tested using the procedures of functional measurement (Anderson, 1970, 1974).

\section{EXPERIMENT 1}

\section{Method}

Ambiguous sentences were shown to subjects one at a time, each accompanied by paraphrases of its two primary interpretations. The subjects' task was to judge the relative likelihood of obtaining one interpretation vs. the other as the meaning of the ambiguous sentence under "normal" conditions.

Stimulus design. Three different 5 by 5 stimulus matrices were constructed based on different ambiguous sentence frames. The three basic sentence frames had different surface structures, but each was ambiguous at the surface-structure level. Each factor of a matrix corresponded to one interpretation of the basic sentence and the levels of each factor were the degrees to which the critical semantic constraint for that interpretation was satisfied.

For example, the first stimulus matrix, the "airport" matrix, consisted of sentences such as: "A cab driver saw a pilot driving 
to the airport." The italicized part of the sentence is the trame, which was constant for the entire matrix. The two noun phrases that are not italicized are the variable parts of the sentence, each of which is associated with one of the two factors of the design. The ambiguity in this sentence frame is that either noun phrase could be the agent of the final verb phrase. The paraphrases of these two interpretations that were shown to the subjects were: "While driving to the airport a cab driver saw a pilot," and "A cab driver saw a pilot who was driving to the airport." For this experiment, each paraphase necessarily contained two variable parts as indicated, and these were identical to the variable parts in the corresponding ambiguous sentence. In the actual stimulus displays, none of the words was italicized, underlined, or emphasized in any way.

For the first interpretation, the initial noun phrase of the ambiguous sentence is the agent of the verb phrase "driving to the airport." Therefore, the five noun phrases that made up the first factor were selected to vary in their compatibility with driving to airports: "A cab driver," "the police," "I," "a boy," and "a dog." For the second interpretation, the second noun phrase of the sentence is the one that is the agent of the verb phrase. Therefore, the five noun phrases that made up the second factor were also selected to vary in their compatibility with driving to airports: "a famous hijacker," "a pilot," "a cab driver," "a boy," and "a dog." The three sentences for which the first and second noun phrases are identical, namely, "a cab driver," "a boy," and "a dog," are syntactically deviant since normally the second occurrence in a sentence of a noun phrase is either made reflexive, if it has the same referrent as the first occurrence, or else is differentiated by some additional modifier, such as: "A cab driver saw another cab driver driving to the airport." Therefore, to avoid spurious effects caused by manipulating syntactic constraints, these three sentences were omitted from the matrix.

The cther two stimulus matrices were constructed in a similar fashion. The "binoculars" matrix used sentences such as: "The girl saw the boy with the binoculars," the paraphrases of which were: "Using the binoculars, the girl saw the boy," and "The girl saw the boy who had the binoculars."

For the first interpretation, the prepositional phrase "with the binoculars" is the instrument of the main verb. Therefore, the verbs that made up the first factor were selected to vary in their compatibility with being done with binoculars: "looked for," "looked at," "saw," "hit," and "touched." For the second interpretation, this prepositional phrase modified the object. Therefore, the noun phrases that made up the second factor were selected to vary in their compatibility with possessing binoculars: "the thief," "the bird-watcher," "the boy," "the gorilla," and "the dog."

The "secretary" matrix consisted of sentences such as: "A good secretary can type quickly written reports," the paraphrases of which were: "A good secretary can quickly type reports which are written," and "A good secretary can type reports which are quickly written."

For the first interpretation, the adverb modifies the verb. Therefore, the verbs that made up the first factor were selected to vary in their compatibility with being done quickly: "type," "find," "file," "correct," and "copy." for the second interpretation, the adverb modifies the adjective of the object. Therefore, the adjectives that made up this factor were selected to vary the compatibility of the actions they described being done quickly: "published," "written," "typed," "filed," and "corrected." The three stimuli for which the verb and the adjective of the object were concerned with the same action (viz., typing, filing, and correcting), violate semantic constraints other than the critical constraints that were meant to be varied. For example, one cannot file reports that are already filed. This means both interpretations become nonsensical independently of the critical semantic constraints governing the adverb. Therefore, these three stimuli were omitted from the matrix
The entire set of stimuli used in Experiment 1 can be seen by referring to Figure 2 .

Apparatus and Procedure. Thirty undergraduate volunteers from the University of California, San Diego, were run individually through one experimental session each. The stimuli were presented one at a time on a video monitor controlled by a PDP-12 computer. The ambiguous sentence was displayed horizontally across the top of the screen and its two interpretations were displayed one at either side, near the bottom of the screen. The two interpretations were randomly assigned to the left and right sides of the screen and the stimulus presentation urder was also randomized and different for each replication for each subject. The judgment responses were made using a graphic rating scale, consisting of a sliding pointer that could be positioned at any point along a $70-\mathrm{mm}$ line. Subjects were instructed to place the pointer so that its position along the line corresponded to the judged relative likelihood of obtaining each of the interpretations from the ambiguous sentence, with each absolute end of the scale defined to be certainty that the corresponding interpretation would be obtained. The pointer was connected to a slide-activated potentiometer so that its position resulted in a voltage level that was monitored by the computer through an analog-to-digital converter. The subject flipped a switch to signal that he had completed positioning the pointer.

After hearing the instructions, subjects judged about 15 of the stimuli while the experimenter answered any questions about the procedure. Then, the experimenter went into an adjacent room and the subject ran through the rest of the total set of stimuli to insure thorough familiarization with the task. This was followed by two more complete replications of the stimuli. Only the data from the last two replications were used in the analysis. Stimulus presentation was self-paced and the entire experimental session lasted about $75 \mathrm{~min}$.

\section{Results and Discussion}

The results of the experiment are shown in Figure 2. In this figure, the data are the filled circles, each of which stands for the judgments to one stimulus averaged over subjects and replications. The curves are the predictions made by the proposed judgment rule when fit to the data using the iterative computer routine STEPIT (Chandler, 1969). Separate predictions were made for each subject, so the curves are the averages of individual subject predictions. The ordinate of the graphs give the judged likelihood that the interpretation obtained would be the one given at the bottom of the graph.

As can be seen from Figure 2, the proposed rule provided a very close fit to the data. The grand root mean squared deviations of the data from the predictions were $.040, .047$, and .042 for the three matrices, respectively. The goodness of fit in the first two matrices provides very strong support for the proposed judgment rule. The good fit of the secretary matrix provided virtually no information for or against the rule, since there were no reliable effects [for rows, $F(4,116)=.80 ;$ for columns, $F(4,116)=1.31 ;$ for interaction. $F(13,377)=1.55]$ in this matrix. Visual inspection of the three graphs does not reveal any simple systematic trends to the deviations of the data from the predictions.

In summary, the proposed judgment rule provides 
30 ODEN

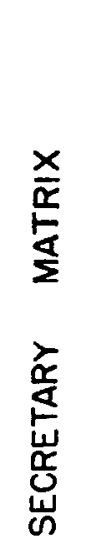

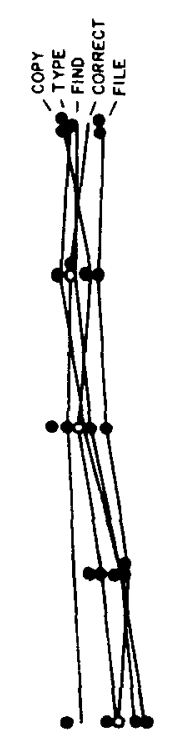

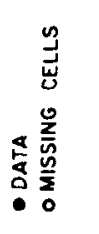

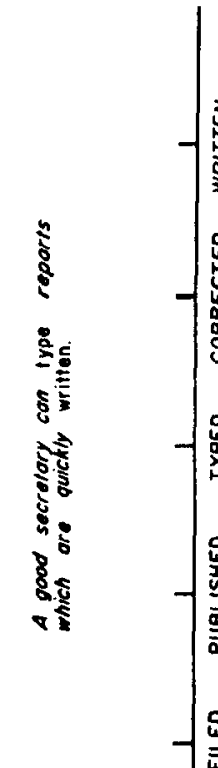

$\mid$

$\frac{\frac{x}{\alpha}}{\frac{1}{a}}$

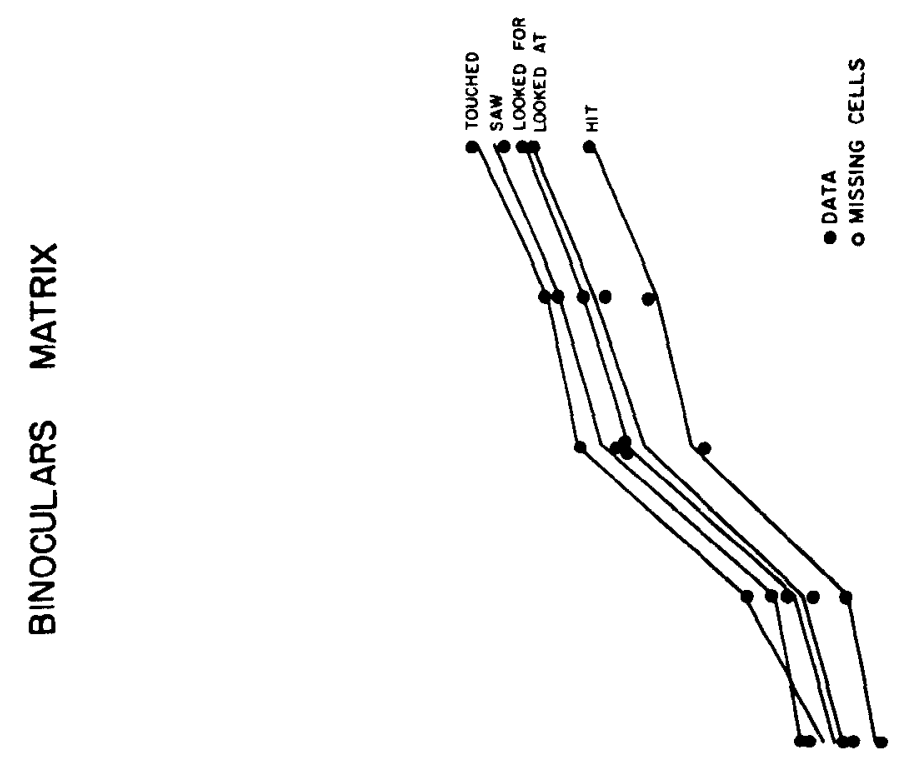

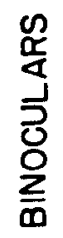

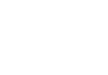

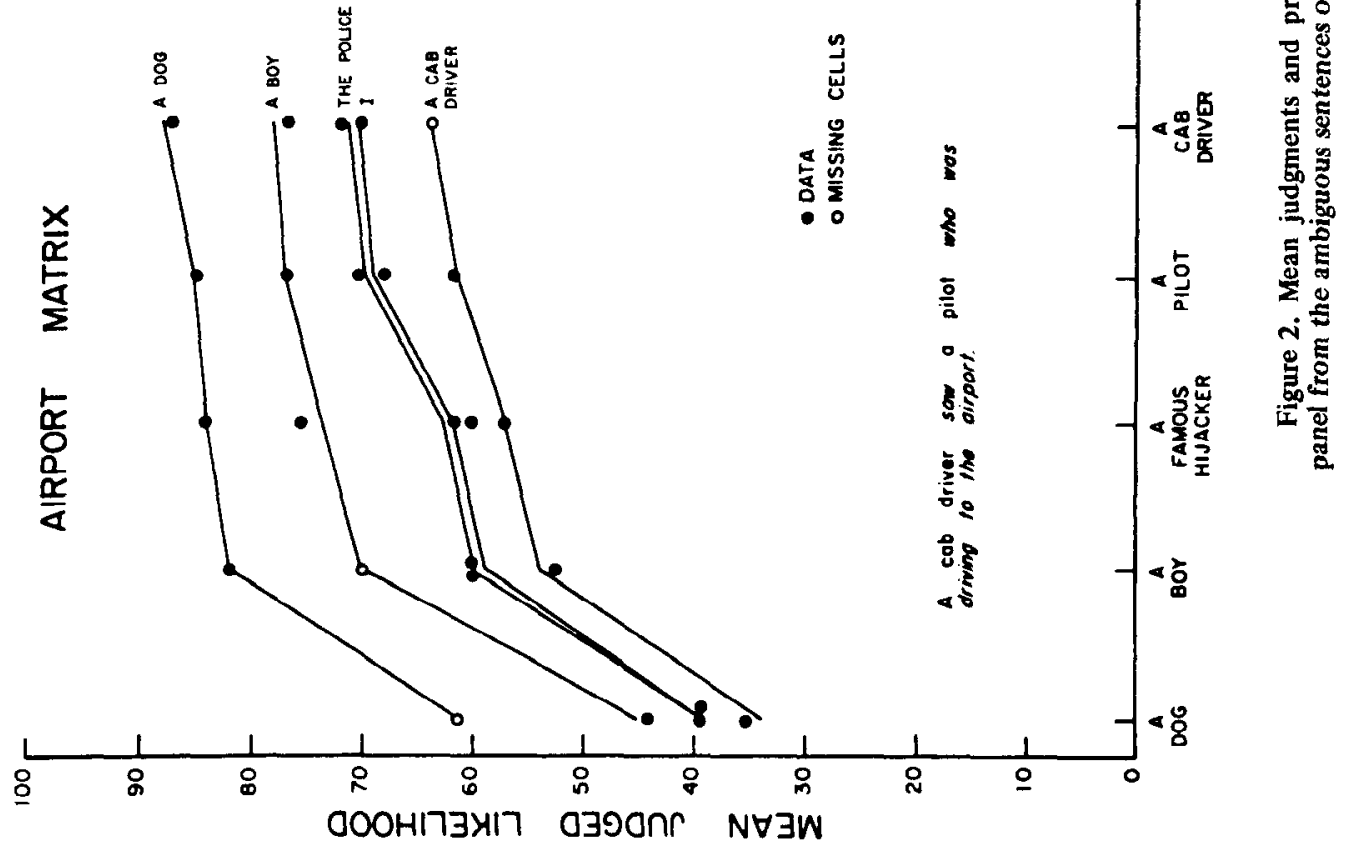


a good account for the data and is, therefore, strongly supported by these results. Consequently, and of greater importance, the results also provide strong support for the psychological reality and independence of semantic constraints as formulated in the Introduction. The judgment rule used a single parameter value to specify the sensibleness of a given interpretation for each of the four or five sentences for which the semantic constraints governing that interpretation were constant. Therefore, the success of the rule in accounting for the data implies that the semantic constraints were stable across these sentences and, therefore, independent of the other semantic constraints that varied from sentence to sentence in the respective row or column of the matrix. Furthermore, this was true for each row and each column of each matrix. Thus, the semantic constraints studied in this experiment have been shown to be relations that the subjects perceive to be separate from the other relations in the sentence and that do not depend upon the total semantic configuration of the sentence. In other words, while continuous semantic constraints may individually be configural (i.e., dependent upon and sensitive to arbitrarily complex patterns of semantic elements), they are also psychologically isolable in that they are evaluated independently of the other semantic and syntactic constraints in the sentence.

The results also clearly establish the necessity of conceptualizing semantic constraints as restrictions that may be more or less satisfied. While not all of the rows and columns are greatly different, there are in both the airport and binoculars matrices several distinct levels of sensibleness on both factors. For example, the sensibleness of a boy having binoculars is between that of a gorilla having binoculars and that of a birdwatcher having binoculars. This is a consistent result, true for all five of the verbs that were used. If it had been the case that semantic constraints could only be either completely satisfied or completely violated, then all of the matrices would be functionally reduced to 2 by 2 designs, and the mean judgment would take on at most four different values. This is clearly not a good description of the obtained data, which display many different levels of judged relative likelihood even at the individual subject level. Thus, semantic constraints provide continuous information about the sensibleness of interpretations.

Details of the method of fitting the model. The judgment rule was fit to the data of each subject for each matrix by the use of the iterative computer routine STEPIT (Chandler, 1969), adapted to run on the PDP.12. This program found the values of the parameters of the rule that minimize the summed squared deviations of the data from the predictions of the rule.

The parameters that provided the best fit to the data for each subject were used to generate the predictions for that subject, and it is these predictions, averaged across subjects, which are plotted in Figure 2. This method of fitting the judgment rule to the data requires that the rule account for the data of each subject individually, rather than simply for the group as a whole. The importance of fitting the individual subjects in assessing the goodness of models is strikingly illustrated by a result of this experiment. In the graph of the binoculars matrix, middle panel of Figure 2, the top two curves cross over each other near the left of the graph. This crossing over of theoretical curves reflects a similar, though somewhat smaller crossover in the data. This crossover of theoretical curves may seem surprising at first, since the proposed judgment rule itself must obey ordinal independence. However, the rule is one of the class of functions whose form is changed by averaging (Estes, 1956). The particular crossover obtained is the result of averaging the predictions made for individual subjects who differed in their ordering of the sensibleness values for certain levels of the matrix. Furthermore, the presence of the theoretical curves crossover indicates that the corresponding empirical crossover in the group data was probably not due to noise nor to a violation of the rule, but instead was most likely merely an artifact of averaging across subjects.

Statistical analysis. A statistical test was performed to assess whether the judgment rule provided a complete account of the data or if there were effects in addition to those accounted for by the rule. This procedure, which is decribed in detail in Leon and Anderson (1974), consisted of performing an analysis of variance [using a missing cells estimation procedure (Bennett \& Franklin, 1954) where required] on the deviations of the data from the predictions. If the rule is correct, then the deviations were just noise and the analysis of deviations should produce no significant effects.

The analysis of the deviations revealed only one statistically significant effect: the interaction in the airport matrix $[F(13,377)=1.76]$. The significant interaction could indicate real effects in the data other than that described by the judgment rule. However, if the deviations from the mean predictions for the airport matrix are plotted, there is no apparent interpretable pattern to this interaction. Therefore, the statistical significance of this one effect probably attests more to the high sensitivity of the analysis than to any important real phenomenon. Thus, the statistical analyses show that, while there are many strong effects in the data, the proposed judgment rule accounts for nearly all of these effects. This provides additional strong support for the validity of the rule and, therefore, of the psychological reality and independence of semantic constraints.

\section{EXPERIMENT 2}

In Experiment 1, the critical semantic constraints were manipulated by varying constrained words 
within the ambiguous sentences. This use of strictly intrasentential manipulations of semantic constraints allowed the stimuli to be very simple, consisting only of the ambiguous sentences themselves. However, intrasentential manipulation increases the difficulty of constructing stimuli that provide a proper test of the judgment rule, since the words within a sentence are usually constrained in many ways for both interpretations, syntactically as well as semantically. This was especially a problem with the secretary matrix and is a major reason for the lack of effects in that matrix: While there are many verbs that are much less compatible with the adverb "quickly" than those actually used, such as "meander," "sleep," "procrastinate," "sneeze," and so forth, it is hard to find ones that are not eliminated by other extraneous constraints of the ambiguous sentence frame. Furthermore, intrasentential manipulation seems to be possible only with surface-structural ambiguities, whereas the hypothesized use of semantic constraints to determine the sensibleness of interpretations should apply to all other kinds of ambiguity as well.

Happily, there are a number of possible solutions to this problem. One approach is to use ambiguous stimuli that have fewer extraneous constraints. For example, it might be easier to construct stimuli consisting of ambiguous noun phrases, such as "ferocious tiger hunter," and "little apple picker," rather than full ambiguous sentences. Another variation on this approach would be to use "telegraphic" stimuli. For example, since newspaper headlines usually leave out many of the syntactic markers, they are less constrained and often ambiguous; for example, "Ancient Indian Remains Uncovered," and "Used Girls to Forge Checks" (from Tempel, 1969). A third solution, which was the one used in the present study, is to manipulate sensibleness by providing information that is semantically related to one of the interpretations but is external to the ambiguous sentence, and therefore not governed by as many of its constraints.

Thus, in Experiment 2 every ambiguous sentence was preceded by a context sentence that was used to manipulate the critical semantic constraints. As an illustration, consider the following sentence from the binoculars matrix of Experiment 1: "The girl looked for the boy with the binoculars." One constraint on the use of binoculars as an optical instrument concerns the distance from the observer to the object: It is more sensible to use binoculars to look for distant objects. This distance is not specified in the sentence above, but could easily be mentioned in a preceding context sentence. Similarly, among the characteristics of the boy that affect the sensibleness of his having binoculars are his age and the activity in which he is involved, both of which may also be given in the context sentence. An example of one stimulus of this type is: "A girl was looking out of her window toward the park across the street where there were a bunch of 3-year-olds playing tag. She was looking for a boy with a pair of binoculars." Notice that it is now easy to vary both the proximity and the age of the children over a wide range. This should produce large effects and, consequently, a stronger test of the judgment rule.

\section{Method}

Subjects were presented with ambiguous sentences preceded by a context of one sentence, along with paraphrases of the two primary interpretations of the ambiguous sentence. The interpretations of each ambiguous sentence were judged as to their likelihood of being obtained from the sentence, in the given context, under normal conditions.

Stimulus design. Three 5 by 5 stimulus matrices were constructed based on different context sentence/ambiguous sentence frames. In this study, the three basic sentence frames were ambiguous at the lexical, surface, and underlying levels, respectively. As in the preceding study, each factor of a matrix corresponded to one interpretation of the ambiguous sentence and the levels of each factor were the degrees to which the critical semantic constraint for that interpretation was satisfied.

The first stimulus matrix, the "accident" matrix, consisted of stimuli such as: "Mary Smith was in a terrible accident which was caused by a child throwing a rock through the car window. She could not bear children after the accident," where again the italicized part of the stimulus is the frame, which was constant for the entire matrix.

The paraphrases of the two interpretations of the ambiguous sentence were: "She was unable to give birth after the accident," and "She could not stand children after the accident." In this experiment, since the ambiguous sentence itself did not change from cell to cell, its two paraphrases were also the same throughout the matrix. The sensibleness of the first interpretation was manipulated by providing information in the context about the agent's probable state of health and, hence, ability to produce children. This was done by stating whether or not she was in an accident and, if so, how serious it was. The sensibleness of the second interpretation was manipulated by providing information about the cause of the accident and the degree to which a child was to blame. Two stimuli for which the agent was not actually in the accident and no child was involved in the cause of the accident were omitted from the matrix, since the ambiguous sentence did not in either sense logically follow from its context.

The second stimulus matrix, the "binoculars II" matrix, consisted of stimuli such as: " $A$ girl was looking out of her window toward a hill a mile away where there were a bunch of 3-year-olds playing tag. She was looking for a boy with a pair of binoculars." The paraphrases of the ambiguous sentence were: "She was using binoculars to look for some boy," and "She was looking for a boy who had a pair of binoculars." The sensibleness of the first interpretation was manipulated by providing information about the distance from the observer to the objects of observation. The sensibleness of the second interpretation was manipulated by providing information about the age and activities of the children.

The third stimulus matrix, the "police" matrix, consisted of stimuli such as: "Center City is sometimes troubled by rioting but the policemen in town are having a big feud. Recently, the mayor ordered the police to stop fighting." The paraphrases of the ambiguous sentence were: "The mayor ordered the police to stop people from fighting," and "The mayor ordered the police to stop fighting with each other." The sensibleness of the first interpretation was manipulated by providing information about the seriousness of fighting going on in town. The sensibleness of the second interpretation was manipulated by providing information about the argumenta- 
tiveness of the police. One of the stimuli, for which the town's problem was vandalism and the characteristic of the police was laziness, was omitted since the ambiguous sentence did not in either sense follow from its context. The entire set of stimuli used in all of the matrices of this experiment can be seen by referring to Figure 3.

Procedure and Apparatus. The procedure was identical to that of Experiment 1 except that at the top of each stimulus display, the ambiguous sentence was preceded by the context sentence. The two sentences were presented in normal prose format with the ambiguous sentence starting where the context sentence left off. All other details of the apparatus and the running of the experiment were the same as in Experiment 1. Subjects were 30 University of California, San Diego, undergraduates, none of whom had been in Experiment 1.

\section{Results and Discussion}

The data and mean predictions are presented in Figure 3, which is of the same form as Figure 2. As can be seen from this figure, the model fits the data very well for all three of the stimulus matrices, with respective grand root mean squared deviations of the data from the model of $.041, .044$, and .049 . Visual inspection does not reveal any simple systematic trends in the deviations for any of the graphs of Figure 3. Thus, the goodness of fit in every case strongly supports the judgment rule and, therefore, also indicates that semantic constraints are psychologically stable, isolable entities.

As hoped, the use of the context sentence to manipulate the semantic constraints allowed greater flexibility in constructing the stimuli and produced consistently larger effects. This put the judgment rule to a stronger test and, therefore, allows more confidence in the conclusions based on its success. Furthermore, the larger effects and wider range of values, especially with the binoculars II matrix, provide even clearer evidence for the continuous nature of semantic constraints and of the sensibleness of interpretations.

Statistical analysis. The method for fitting the judgment rule to the data was the same as that of Experiment 1 . The analysis of variance on the deviations of the data from the predictions was also performed and none of the effects for any of the matrices was statistically significant. Thus, while there were strong effects in the raw data to be accounted for, the fact that there were none left in the analysis of deviations provides very strong support for the judgment rule and, consequently, very strong support for the proposed formulation of semantic constraints.

\section{GENERAL DISCUSSION}

The basic conclusions to be drawn from these studies are (1) that people use the relative sensibleness of the interpretations of an ambiguous sentence in judging which meaning they feel they would be likely to obtain for the sentence, (2) that semantic constraints are used to determine the degree of sensibleness of each interpretation, and (3) that these semantic constraints are continuous restrictions that are psychologically independent and stable across the various sentences in which they occur. The emphasis in each of these statements is on the continuousness of the semantic constraints and of the sensibleness values they determine. In their discrete form, these conclusions have been more or less generally accepted for traditional all-or-none conceptions of semantic constraints (e.g., Katz \& Fodor, 1963), but it was by no means obvious that they would be found to be true when semantic constraints were generalized to the continuous case. For example, with discrete semantic constraints, independence simply means that changing one constraint does not cause another to switch from the state of being satisfied to the state of being not satisfied, or vice versa. In contrast. with continuous semantic constraints, independence is a more stringent requirement that does not even allow changes in the degree to which constraints are satisfied as a result of the condition of other constraints. Similarly, while it has always been apparent that an interpretation must make sense in order for it to be chosen as the meaning of an ambiguous sentence, it was not at all certain that subjects would make use of the full information about the degree of sensibleness of each interpretation in making their judgments.

\section{Determining the Bias of Ambiguous Sentences}

As was mentioned in the Introduction, the judgment task used in the present experiments has been used previously (e.g., Olson \& MacKay, 1974) to measure the bias of ambiguous sentences. As such, the judgment task is a distinct improvement over the somewhat more common procedure of simply using the proportion of subjects who choose one interpretation vs. the other when given a forced choice. However, in the previous uses of the judgment task, the responses have been averaged over subjects, which makes it difficult to interpret what a given bias measure signifies. For example, a value of .5 could result from half of the subjects totally preferring one interpretation and half totally preferring the alternative, or it could result from all of the subjects being completely ambivalent, and/or it could be due to any number of mixtures. These different patterns could be expected to have greatly different consequences for any language processing task that is affected by the bias of ambiguous sentences.

The results of the present experiments have a number of ramifications for this problem of determining bias. First of all, the functional measurement procedures (Arderson, 1970, 1974) used in the present experiments scaled and validated the sensibleness values for each subject individually. Since the subjects' judgments were found to be a direct function of the relative sensibleness, this means that reliable estimates of bias may be obtained in this manner for each individual 


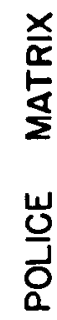
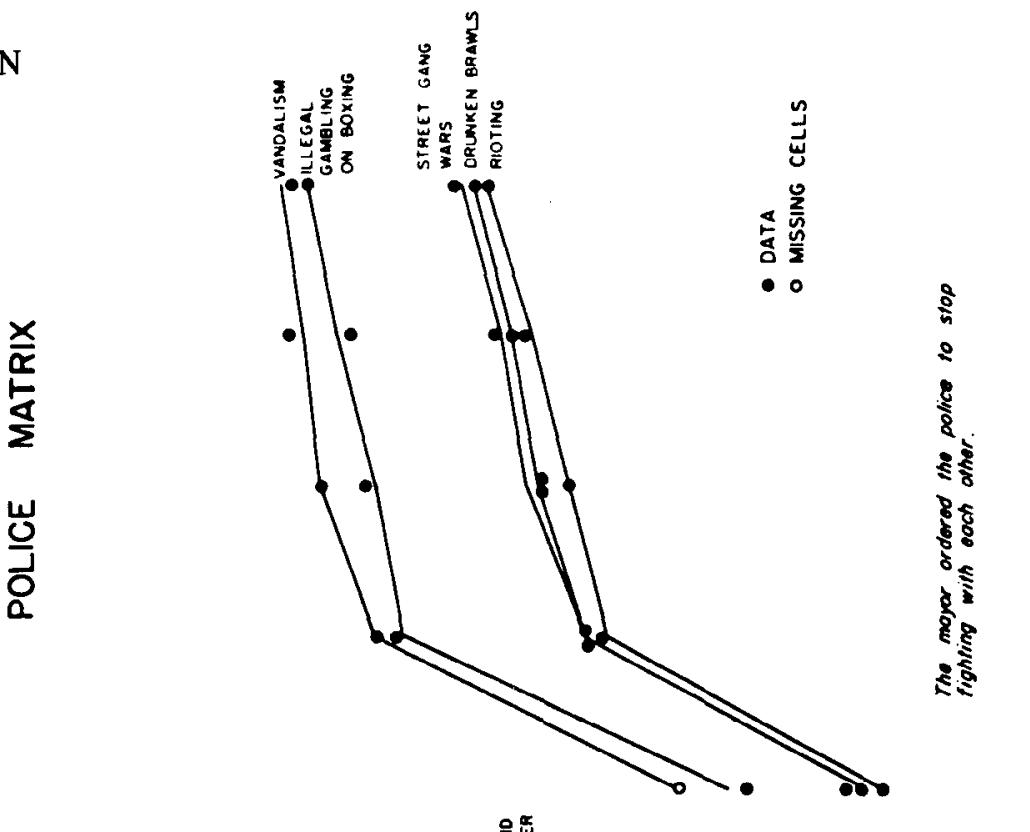

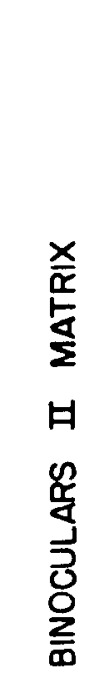

$-1$

-

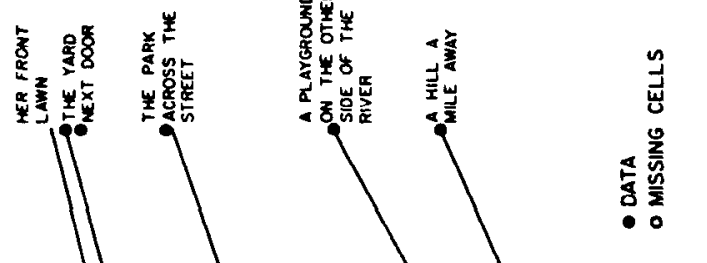

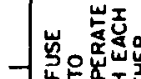

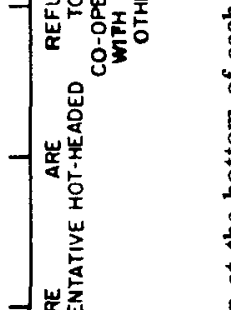

.
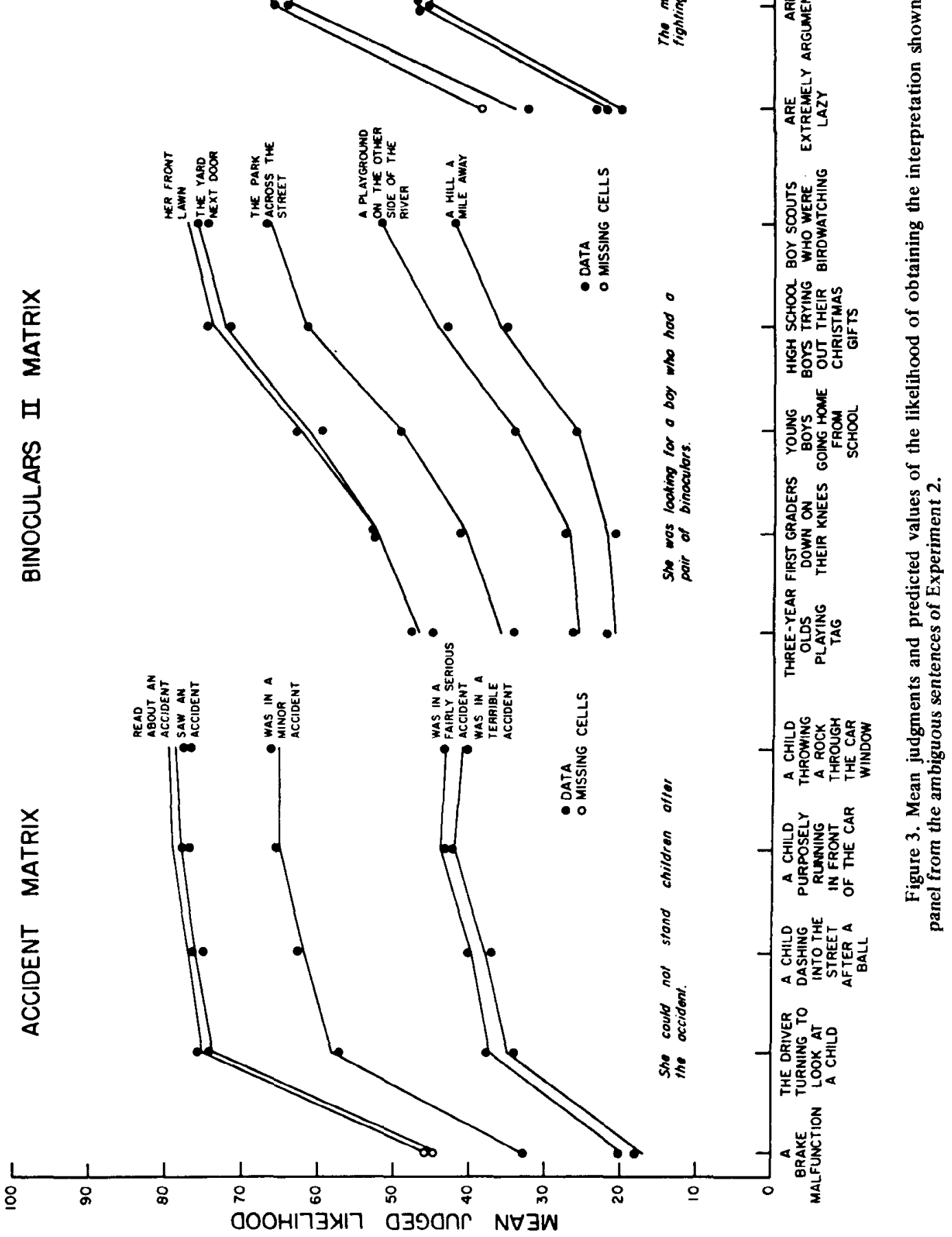
subject for each sentence. This would allow a very precise determination of the effects of bias on language processing at the individual subject level.

Second, the present results illustrate that it is possible to "tune" ambiguous sentences to any of a number of degrees of bias by manipulating the sensibleness of the alternative interpretations. In this way, quite exact values of bias may be obtained when this is used as the independent variable, and, therefore, this technique could be very valuable in studying specifically how bias affects language processes.

Third, the present results suggest that the importance of bias in processing ambiguous sentences may actually be in large part due to the effects of the sensibleness of the individual interpretations. Extending this argument, it may be that a given bias will have different effects depending upon the particular sensibleness values of the separate interpretations. For example, a sentence with interpretations that are .9 and .3 sensible for a given subject might behave in a systematically different way from a sentence with interpretations that are .3 and .1 sensible, even though the bias would be .75 for both sentences, assuming everything else remains constant. If this is the case, then the sensibleness of each interpretation should be considered instead of or along with the bias.

Central to each of these approaches to studying and measuring bias is the fact that each of the matrices used in the present experiments was built around a single ambiguous sentence and constituted, in essence, a complete, self-contained experiment. That is, each matrix in and of itself provided a separate and complete test of the judgment rule. This is one of the major advantages of the procedure used in these studies, that it provides single-sentence tests of the hypothesized processes. Such tests insure that the obtained results are not merely artifacts produced by averaging over many diverse kinds of sentences, each of which might individually produce quite different results. Of course, there are many types of psychological tasks for which it is preferable not to include too many sentences that are similar. However, even with such tasks, it is nearly always possible to perform the judgment task on full matrices of sentences after the other tasks, in order to obtain the precise measurements of bias or of the sensibleness of individual interpretations.

Where feasible, the use of "single-sentence designs" can be a very powerful tool. Linguists have long been aware of the necessity for their theories to account for individual sentences rather than just for general classes of sentences. This is also true for psycholinguistics: There are too many individual differences from sentence to sentence to justify a complete reliance on group analyses. This argument is exactly analogous to that often made (e.g., Estes, 1956) about the virtues of performing single-subject analyses of exneriments. With single-sentence designs, it is still important, of course, to replicate results with several sets of stimuli, just as it is important to replicate results with new groups of subjects. In the present experiments, the results have been established for five different singlesentence designs using lexical, surface-structural, and underlying structural ambiguities and two different methods for manipulating the semantic constraints.

\section{How Continuous Semantic \\ Constraints Might Be Used}

According to Bever, Garrett, and Hurtig (1973), when an ambiguity is encountered during language comprehension, both interpretations are processed until the end of the clause is reached, at which point one of the interpretations is chosen to be the meaning of the sentence. Bever et al. (1973) do not explain how this choice is made but, given the results of the present experiments, a natural hypothesis would be that the relative sensibleness of the interpretations is used in making this choice. The present experiments do not directly address this hypothesis, since the judgmental task differs in significant ways from natural language comprehension, particularly in allowing subjects unlimited time and in making them fully aware of the ambiguity. However, the results of a recently completed experiment (Oden \& Ekberg, Note 2) indicate that the relative sensibleness of interpretations as specified by semantic constraints does actually determine which interpretation is obtained during normal comprehension in a paraphrasing task. Thus, it is reasonable to begin to develop language processing models that use the continuous sensibleness information provided by semantic constraints. Some of the details of such a system have been proposed by Oden (1974) and are summarized here.

First of all, the component processors (e.g., syntactic, semantic) of the language comprehension system are assumed to be concurrent in operation and mutually contingent. That is, they operate simultaneously, each performing whatever analysis can be done using the information available to it at any given point in time, including the information provided by each of the other processors (c.f. Marslen-Wilson, 1975; Reddy, Erman, \& Neely, 1973; Rumelhart, Note 3). In such a system, the semantic processor can build tentative semantic structures during the course of language processing and can also apply the relevant semantic constraints to each of these structures to determine their degree of sensibleness.

This building and evaluating of candidate semantic structures is assumed to take place in parallel for all possible interpretations, as proposed by the hypothesis of Bever et al. (1973) and supported by the results of numerous experiments (e.g., Foss, 1970; Foss \& Jenkins, 1973; Holmes et al., 1977; Lackner \& Garrett, 1972; MacKay, 1966; Mistler-Lachman, 1972; Swinney \& Hakes, 1976). However, the proposed 
system also includes two mechanisms that use the degree-of-sensibleness information to eliminate interpretations, so that it is not necessary to process all of them completely. The first mechanism, absolute judgment, rejects a candidate interpretation whenever its sensibleness value falls below some cutoff point. Since the cutoff seems to be adjustable, so that, for example, people will accept quite nonsensical sentences from a young child or Lewis Carroll, a continuous measure of sensibleness is required even for absolute judgment. The second mechanism, relative judgment, eliminates all but the most sensible interpretation, but only after enough processing has been performed (e.g., at the end of a clause) so that the system has an accurate assessment of the relative sensibleness of the interpretations.

Thus, the proposed system would strongly exploit the rich source of information provided by continuous semantic constraints. Many experiments will be required to evaluate a system of this complexity, but the evidence for continuous semantic constraints obtained in the present studies provides a promising beginning for this endeavor.

\section{REFERENCE NOTES}

1. Oden, G. C., \& Hogan, M. E. A fuzzy propositional model of negation on semantic continua. Paper presented at the meetings of the Midwestern Psychological Association, Chicago, May 1977.

2. Oden, G. C., \& Ekberg, K. Semantic constraints determine the relative sensibleness of interpretations of ambiguous sentences. Unpublished manuscript, 1977.

3. Rumelhart, D. E. Toward an interactive model of reading (Technical Report No. 56). San Diego: University of California, Center for Human Information Processing, March 1976.

\section{REFERENCES}

ANderson, J. R. Language, memory, and thought. Hillsdale, N.J: Lawrence Erlbaum, 1976.

ANDERSon, N. H. Functional measurement and psychophysical judgment. Psychological Review, 1970, 77, 153-170.

ANDERSON, N. H. Information integration theory: A brief survey. In D. H. Krantz, R. C. Atkinson, R. D. Luce, \& P. Suppes (Eds.), Contemporary developments in mathematical psychology (Vol. 2). San Francisco: W. H. Freeman, 1974.

BenNett, C. A., \& Franklin, N. L. Statistical analysis in chemistry and the chemical industry. New York: Wiley, 1954.

Bever, T. G., Garrett, M. F., \& Hurtig, R. The interaction of perceptual processes and ambiguous sentences. Memory \& Cognition, 1973, 1, 277-286.

CAIRNs, H. S. Effects of bias on processing and reprocessing of lexically ambiguous sentences. Journal of Experimental Psychology, 1973, 97, 337-343.

Chander, J. P. Subroutine STEPIT-Finds local minima of a smooth function of several parameters. Behavioral Science, 1969, 14, 81-82.

EsTES, W. K. The problem of inference from curves based on group data. Psychological Bulletin, 1956, 53, 134-140.
Foss, D. J. Some effects of ambiguity upon sentence comprehension. Journal of Verbal Learning and Verbal Behavior, 1970, 9, 699-706.

Foss, D. J., \& Jenkins, C. M. Some effects of context on the comprehension of ambiguous sentences. Journal of Verbal Learning and Verbal Behavior, 1973, 12, 577-589.

Hersh, H. M., \& Caramazza, A. A fuzzy set approach to modifiers and vagueness in natural language. Journal of Experimental Psychology: General, 1976, 105, 254-276.

Holmes, V. M., Arwas, R., \& Garrett, M. F. Prior context and the perception of lexically ambiguous sentences. Memory \& Cognition, 1977, 5, 103-110.

KATZ, J. J., \& FoDor, J. A. The structure of a semantic theory. Language, 1963, 39, 170-210.

Kintsch, W. The representation of meaning in memory. Hillsdale, N.J: Lawrence Erlbaum, 1974.

LACKNER, J. R., \& GARRETT, M. F. Resolving ambiguity: Effects of biasing context in the unattended ear. Cognition, 1972, 1, 359-372.

LAKOFF, G. HEDGES: A study in meaning criteria and the logic of fuzzy concepts. Papers from the eighth regional meeting of the Chicago Lingusitic Society. Chicago: University of Chicago Linguistic Department, 1972.

LeON, M., \& ANDERson, N. H. A ratio rule from integration theory applied to inference judgments. Journal of Experimental Psychology, 1974, 102, 27-36.

Mackay, D. G. To end ambiguous sentences. Perception \& Psychophysics, 1966, 1, 426-436.

MacKay, D. G. Mental diplopia: Towards a model of speech perception at the semantic level. In G. B. Flores d'Arcais \& W. J. M. Levelt (Eds.), Advances in psycholinguistics. Amsterdam: North-Holland, 1970.

Marsten-Wilson, W. D. Sentence perception as an interactive parallel process. Science, 1975, 189, 226-228.

Mistler-Lachman, J. L. Levels of comprehension in processing of normal and ambiguous sentences. Journal of Verbal Learning and Verbal Behavior, 1972, 11, 614-623.

Norman, D. A., \& Rumel.hart, D. E. (Eds.). Explorations in cognition. San Francisco: Freeman, 1975.

Oden, G. C. Semantic constraints and ambiguity resolution. (Doctoral dissertation, University of California, San Diego, 1974.) Dissertation Abstracts International, 1975, 35, 4227-B. (University Microfilms No. 75-4651.)

Oden, G. C. Fuzziness in semantic memory: Choosing exemplars of subjective categories. Memory \& Cognition, 1977, 5, 198-204.

ODEN, G. C. Integration of fuzzy logical information. Journal of Experimental Psychology: Human Perception and Performance, in press.

ODEN, G. C., \& ANDERson, N. H. Integration of semantic constraints. Journal of Verbal Learning and Verbal Behavior, 1974, 13, 138-148.

Olson, J. N., \& MACKAY, D. G. Completion and verification of ambiguous sentences. Journal of Verbal Learning and Verbal Behavior, 1974, 13. 457-470.

Reddy, D. R., Erman, L. D., \& Neely, R. B. A model and a system for machine recognition of speech. IEEE Transactions on Audio and Electroacoustics, 1973, AU-21, 229-238.

Rosch, E. H. On the internal structure of semantic and perceptual categories. In T. E. Moore (Ed.), Cognitive development and the acquisition of language. New York: Academic Press, 1973.

Rosch, E. H. Cognitive representations of semantic categories. Journal of Experimental Psychology: General, 1975, 104, 192-233.

Swinney, D. A., \& Hakes, D. T. Effects of prior context upon lexical access during sentence comprehension. Journal of Verbal Learning and Verbal Behavior, 1976. 15, 681-689. 
TEMPEL, E. Humor in the headlines. New York: Pocket Books, 1969.

ZADEh, L. Fuzzy sets. Information and Control, 1965, 8, 338-353.

ZADEH, L. A. Fuzzy logic and its application to approximate reasoning. Information processing 74: Proceedings of the
IFIP Congress 74. North Holland: Amsterdam, 1974. Pp. 591-594.

(Received for publication August 18, 1977; accepted September 30, 1977.) 